\title{
Acute effect of propranolol and isosorbide-5-mononitrate administration on renal blood flow in cirrhotic patients
}

\author{
A J Stanley, I A D Bouchier, P C Hayes
}

\begin{abstract}
Background-Propranolol and isosorbide5-mononitrate (ISMN) are increasingly used in the prophylaxis of variceal haemorrhage in cirrhosis. However, recent studies have suggested that these drugs may compromise renal function, possibly by reducing renal blood flow.

Aims-To assess the acute effects of propranolol and ISMN on renal blood flow and other haemodynamic parameters in cirrhosis.

Patients and methods-Twenty six cirrhotic patients were given either $80 \mathrm{mg}$ propranolol, $20 \mathrm{mg}$ ISMN, or a combination of the two drugs. Unilateral renal blood flow (RBF), azygos blood flow (AZBF), hepatic venous pressure gradient (HVPG), mean arterial pressure (MAP), and heart rate (HR) were recorded prior to and one hour after drug administration.

Results-Propranolol caused a reduction in HR $(p<0.005)$, AZBF $(p<0.01)$, and HVPG $(p=0.05)$, but no change in MAP or RBF (454.1 (77.3) versus $413.9 \quad(60.3)$ $\mathrm{ml} / \mathrm{min})$. ISMN reduced MAP $(p<0.005)$ and HVPG $(p<0.01)$, but had no effect on HR, AZBF, or RBF (302.5 (49.4) versus $301.7(58.8) \mathrm{ml} / \mathrm{min})$. Combined treatment reduced MAP $(p<0.005)$, AZBF $(p<0.05)$, and HVPG $(p=0.002)$, but $H R$ and RBF (419.2 (62.6) versus $415.1(61.1) \mathrm{ml} / \mathrm{min})$ remained unchanged.

Conclusions-Despite the anticipated changes in other haemodynamic parameters, acute propranolol and/or ISMN administration did not reduce RBF. These drugs do not seem to compromise RBF in cirrhosis.
\end{abstract}

(Gut 1998;42:283-287)

Keywords: cirrhosis; portal hypertension; renal blood flow; propranolol; nitrates

Department of

Medicine, Royal

Infirmary of

Edinburgh,

Lauriston Place,

Edinburgh EH3 9YW,

UK

A J Stanley

I A D Bouchier

P C Hayes

Correspondence to: Dr Stanley.

Accepted for publication 5 September 1997 tor that reduces portal pressure and hepatic vascular resistance. ${ }^{6-8}$ It has been shown to reduce the risk of variceal bleeding, ${ }^{910}$ and can be used as an alternative treatment for patients with portal hypertension who are intolerant to $\beta$ blockade. More recently, reports have suggested that the combination of propranolol and ISMN may be the optimum treatment to reduce portal pressure and overcome the problem of non-response to propranolol. ${ }^{11}{ }^{12}$

Several recent studies have suggested that $\beta$ blockers and particularly nitrates may compromise renal function in cirrhosis, possibly due to a reduction in renal perfusion secondary to the associated reduced systemic blood pressure. $^{12-17}$ The aim of our study was to investigate the effect of propranolol, ISMN, and the combination of both drugs on renal blood flow and systemic and splanchnic haemodynamics in cirrhotic patients.

\section{Patients and methods}

Twenty six patients ( 12 female, mean age 51.1 years (range 33-70), mean Child-Pugh score (CPS) $9.2(0.6))$ with cirrhosis were studied. Eighteen patients had ascites at the time of study which was clinically graded as mild, moderate, or severe. Diagnosis of cirrhosis was based on liver biopsy (20 patients) or the presence of chronic liver biochemical abnormalities and endoscopically proven varices (six patients). Cirrhosis was alcohol related in 21 patients, and secondary to primary biliary cirrhosis in two, primary sclerosing cholangitis in one, sarcoid in one, and $\alpha_{1}$ antitrypsin deficiency in one. No patient had biochemical evidence of renal dysfunction prior to study (serum urea greater than $6.6 \mathrm{mmol} / 1$ or serum creatinine greater than $150 \mu \mathrm{mol} / \mathrm{l}$ ) and none was receiving vasoactive medication at the time of study. In addition, no patient had suffered a gastrointestinal haemorrhage within the previous four months. All patients gave informed consent and the study was approved by the Lothian Ethics Committee.

Studies were undertaken in the Department of Medicine catheter laboratory, on fasted patients in the supine position. Vascular catheters were positioned under fluoroscopic guidance via an 8.5 FG right femoral venous introducer (Baxter Healthcare Corporation, USA), inserted after local infiltration with $2 \%$ lignocaine. A balloon catheter (Sidewinder II, Cordis Corporation, USA) was inserted through the introducer to record both the free (FHVP) and wedged (WHVP) hepatic venous pressure. Hepatic venous pressure gradient (HVPG) was calculated as WHVP minus FHVP.

A double thermister, reverse thermodilution catheter (Webster Laboratories, California, USA) was then inserted through the introducer 
Table 1 Patient characteristics

\begin{tabular}{|c|c|c|c|}
\hline & Propanolol $(n=9)$ & $\operatorname{ISMN}(n=8)$ & $\begin{array}{l}\text { Combined treatment } \\
(n=9)\end{array}$ \\
\hline Age (y) & $52.7(2.3)$ & $50.5(2.8)$ & $51.1(1.9)$ \\
\hline Child-Pugh score & $9.2(1.0)$ & $9.4(1.5)$ & $9.1(1.1)$ \\
\hline $\begin{array}{l}\text { No with ascites (mild or } \\
\text { moderate/severe) }\end{array}$ & $5 / 0$ & $4 / 1$ & $7 / 1$ \\
\hline $\begin{array}{l}\text { Serum creatinine }(\mu \mathrm{mol} / \mathrm{l}) \text {, } \\
\text { mean (range) }\end{array}$ & $83.1(63-136)$ & $92.1(65-142)$ & $88.8(66-126)$ \\
\hline Aetiology (ALD/other) & $8 / 1$ & $6 / 2$ & $7 / 2$ \\
\hline
\end{tabular}

ALD, alcoholic liver disease.

and positioned in the left renal vein. The proximal thermister was confirmed to be within the lumen of the renal vein by injection of $5 \mathrm{ml}$ dilute contrast media (Conray 280, May \& Baker Ltd, Dagenham, UK). Recordings of unilateral renal blood flow (RBF) were made using a custom built interface (B55724 type CF) and an IBM microcomputer processor (PS2-286) as previously reported. ${ }^{18}$ Mean blood flows were recorded over a 30 second period with the patient breathing normally at rest.

Finally, the reverse thermodilution catheter was positioned in the azygos vein and azygos blood flow (AZBF) recorded in a similar manner to RBF. The catheter was then left in position for the duration of the study.

When recruited for the study, patients were matched for age and CPS for each therapeutic regimen (table 1). Nine patients were then given $80 \mathrm{mg}$ oral propranolol, eight patients 20 mg oral ISMN, and nine patients both treatments. One hour later AZBF was again recorded, then the reverse thermodilution catheter was manouevred back into the initial position in the left renal vein and repeat $R B F$ measurement made as described above. Finally, repeat measurements of the WHVP and FHVP were recorded using the balloon catheter. The sheath was then removed and firm pressure applied until haemostasis was ob- tained. Heart rate (HR) and mean arterial pressure (MAP) were also recorded for the duration of the study and for four hours thereafter. In all patients, the duration of the study was less than 3.5 hours.

STATISTICAL ANALYSIS

Results are expressed as mean (SEM) or range where indicated. For parametric data, the paired Student's $t$ test and Pearson's correlation were used. The Wilcoxon signed ranked test and Kendall correlation were used for non-parametric variables. A $p$ value of less than 0.05 was taken to be significant.

\section{Results}

All patients completed the study without difficulty and no side effects were observed or reported. There was no statistical difference between the three therapeutic groups with regard to baseline RBF or other haemodynamic parameters. Baseline RBF did not correlate with CPS, HVPG, or ascites severity. Table 2 shows changes in HR and MAP for each treatment group at one hour following medication.

Following administration of propranolol, there was a fall in HVPG from 16.3 (2.2) to 12.9 (1.9) $\mathrm{mm} \mathrm{Hg}(\mathrm{p}=0.05)$ and in AZBF from 471.5 (85.8) to $293.4(42.6) \mathrm{ml} / \mathrm{min}(\mathrm{p}<0.01)$ (fig 1). There was no effect on RBF (454.1 (77.3) versus $413.9(60.3) \mathrm{ml} / \mathrm{min}$ ) (fig 2).

ISMN treatment led to a fall in HVPG from 14.9 (1.9) to 11.0 (1.3) $\mathrm{mm} \mathrm{Hg}(\mathrm{p}<0.01)$, but had no effect on either AZBF (573.4 (183.1) versus 576.0 (145.1) $\mathrm{ml} / \mathrm{min}$ ) (fig 1) or $\mathrm{RBF}$ (302.5 (49.4) versus $301.7(58.8) \mathrm{ml} / \mathrm{min}$ ) (fig 2).

Following administration of the combination of propranolol and ISMN, HVPG fell from $15.1(1.8)$ to $8.9(1.1) \mathrm{mm} \mathrm{Hg}(\mathrm{p}=0.002)$ and AZBF fell from 612.5 (187.6) to 358.2 (57.0)

Table 2 Systemic haemodynamic effects

\begin{tabular}{|c|c|c|c|c|c|c|}
\hline & \multicolumn{2}{|l|}{ Propanolol } & \multicolumn{2}{|l|}{$I S M N$} & \multicolumn{2}{|c|}{ Combined treatment } \\
\hline & Before & After & Before & After & Before & After \\
\hline HR (bpm) & $81.6(3.9)$ & $71.7(4.2)^{\star}$ & $86.8(3.5)$ & $91.0(3.3)$ & $79.9(4.8)$ & $71.4(4.4)$ \\
\hline $\mathrm{MAP}(\mathrm{mm} \mathrm{Hg})$ & $82.6(3.4)$ & $78.2(3.3)$ & $84.5(3.3)$ & $76.9(3.6)^{\star}$ & $78.1(4.6)$ & $68.7(3.4)^{\star}$ \\
\hline
\end{tabular}

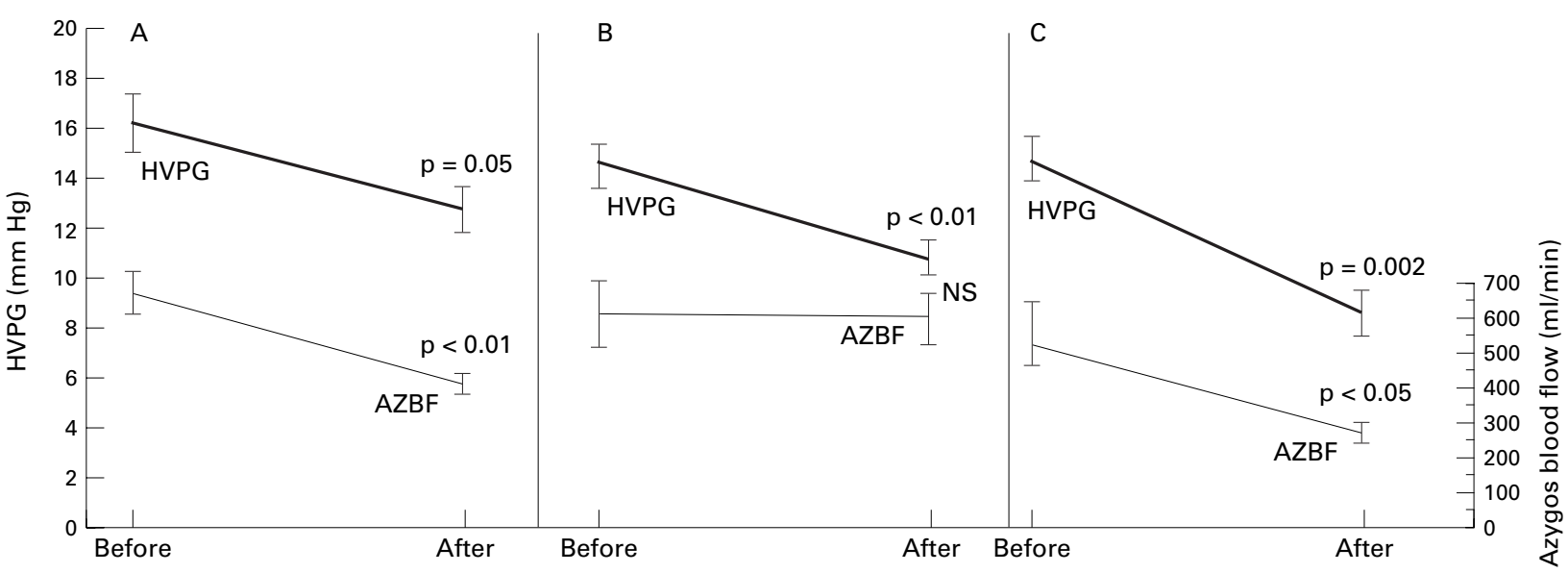

Figure 1 Changes in HVPG and AZBF one hour after a single dose of $(A)$ propranolol, (B) ISMN, or (C) combined treatment. 


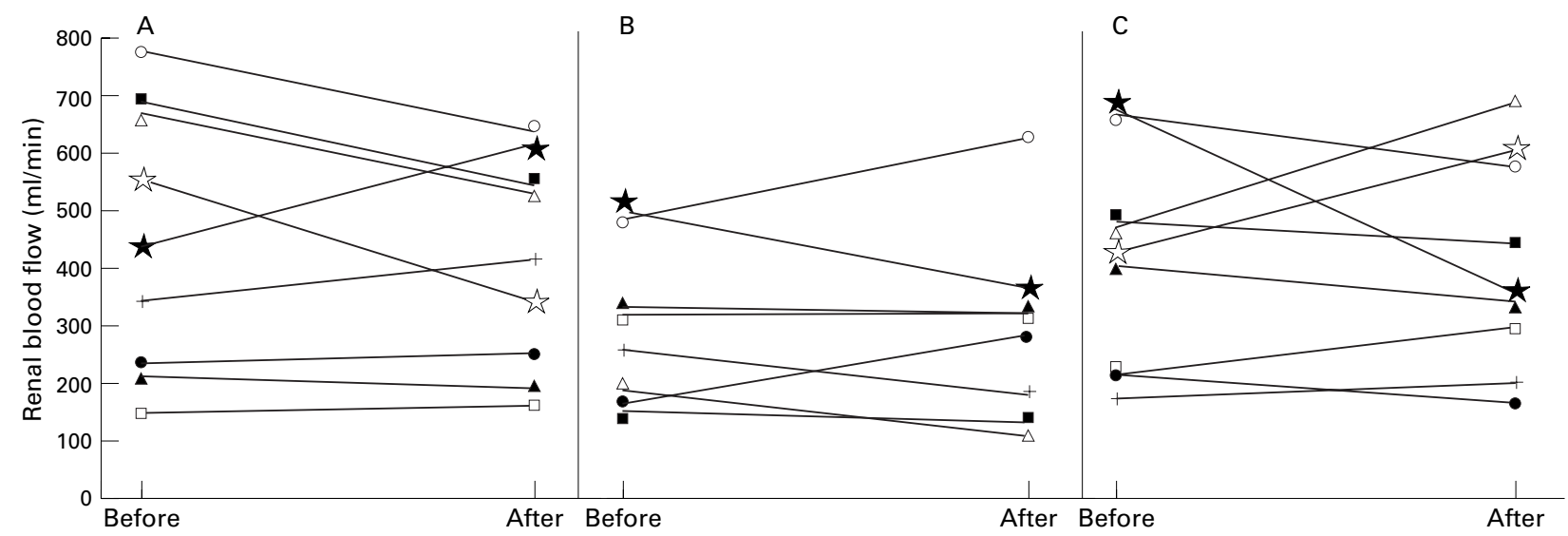

Figure 2 Unilateral RBF one hour after a single dose of (A) propranolol, (B) ISMN, or (C) combined treatment.

$\mathrm{ml} / \mathrm{min}(\mathrm{p}<0.05)$ (fig 1). Again, there was no effect on RBF (419.2 (62.6) versus 415.1 (61.1) $\mathrm{ml} / \mathrm{min}$ ) (fig 2).

When the 18 patients with ascites were considered alone, there remained no effect on $\mathrm{RBF}$ of any treatment when these patients were grouped together, or when analysed in treatment subgroups.

\section{Discussion}

We undertook this study to assess the acute effect of propranolol, ISMN, and the combination of both drugs on RBF in cirrhotic patients. Despite the anticipated changes in other haemodynamic parameters, no change in $\mathrm{RBF}$ was detected.

Any reduction in renal function is critical in cirrhotic patients, because they are already at risk of renal impairment due to reduced RBF, glomerular filtration rate (GFR), and sodium and water excretion. ${ }^{19}$ Recent suggestions that $\beta$ blockers and nitrates may have deleterious renal effects in cirrhosis include reports of reduced renal sodium excretion ${ }^{121316}$ and reduced renal perfusion ${ }^{14-17}$ following administration of these drugs. This is important in view of their increasing use as prophylaxis against variceal haemorrhage..$^{1-5} 910$

Generally, autoregulation in the kidney maintains a constant $\mathrm{RBF}$ over a wide range of arterial blood pressures. ${ }^{20}$ Henriksen and RingLarsen suggest that in cirrhosis, a pharmacologically induced fall in MAP may compromise renal perfusion and GFR, leading to a subsequent reduction in sodium and water excretion, partly due to a shift in this autoregulation. ${ }^{17}$

$\beta$ Blockers are the most widely used drugs in the prophylaxis of variceal haemorrhage. Most investigators have studied propranolol in this role, although nadolol has been used by some researchers. In addition to their portal hypotensive action, these drugs reduce cardiac output and increase systemic vascular resistance but have less effect on MAP. ${ }^{21}$

The renal effects of $\beta$ blockers are complex due to their haemodynamic and neurohumoral actions. Propranolol treatment leads to increased circulating concentrations of noradrenaline which can cause sodium and water retention ${ }^{22}$; however, reduced levels of renin, angiotensin, and aldosterone $\left(\beta_{1}\right.$ blockade) are also observed which increase salt and water excretion. ${ }^{23}$ In addition, renal vasoconstriction due to $\beta_{2}$ blockade has been suggested as a mechanism by which $\beta$ blockers may impair $\mathrm{RBF}$ and GFR. ${ }^{20}$ However, the clinical significance of these changes remains unclear and data on cirrhotic patients are limited.

$\beta$ Blockers have been shown to reduce the frusemide stimulated increase in renal interlobular arterial flow, ${ }^{14}$ and reduce estimated RBF in cirrhosis as assessed by Doppler ultrasonography. ${ }^{15}$ However, Bataille et al found no effect on RBF of acute propranolol treatment when given to cirrhotic patients, ${ }^{24}$ and no difference in creatinine clearance was detected between cirrhotic patients given diuretics alone and those given propranolol and diuretics. ${ }^{13}$ In addition, propranolol has been reported as having no effect on GFR in cirrhosis despite suppressing renin secretion, ${ }^{25}$ and Bernardi et al found that propranolol actually increased GFR and natriuresis in ascitic cirrhotics with high sympathetic tone. ${ }^{23}$ Other studies have reported both decreased ${ }^{13}$ and increased $^{26}$ natriuresis in cirrhotic patients in response to propranolol.

Up to $40 \%$ of cirrhotic patients do not have a reduction in portal pressure in response to propranolol despite a fall in AZBF and are termed "non-responders". In addition, approximately $15 \%$ of patients are intolerant of the drug. ${ }^{27}{ }^{28}$ Therefore, nitrates are commonly used as an alternative treatment, or in combination with propranolol.

Nitrates are venodilators that reduce cardiac output and MAP in cirrhosis. ${ }^{7} 1629$ They are effective in reducing portal pressure and can convert propranolol non-responders into responders. This is consistent with our findings of only a mild reduction in HVPG following propranolol, but a significant reduction following ISMN and particularly the combination treatment. Recent studies have suggested that the combination of ISMN and $\beta$ blockers may be the optimum treatment in portal hypertension. ${ }^{112}$

There has been concern that nitrates may compromise renal function in patients with advanced liver disease, due to the associated fall in blood pressure and activation of the 
sympathetic and renin-angiotensin-aldosterone systems. Vorobioff et al found that $57 \%$ of cirrhotic patients with ascites or a history of ascites developed worsening of ascites when given propranolol and nitrates, compared with no worsening of ascites in any patient given propranolol alone. ${ }^{12}$ ISMN has also been shown to reduce renal plasma flow, GFR, and sodium and water excretion and increase renin and aldosterone values in cirrhosis. ${ }^{16}$ The fall in GFR and free water clearance was greater in those patients who had ascites.

However, Morrillos et al found no effect on inulin clearance, free water clearance, plasma renin activity, aldosterone concentration, or ascites outcome in cirrhotic patients given long term propranolol and ISMN, despite a reduction in blood pressure. ${ }^{30}$ In addition, Merkel et al found no deterioration in renal function in cirrhotic patients given six months of ISMN and nadolol compared with patients given nadolol alone. ${ }^{31}$ Although there was a correlation between the fall in blood pressure and the rise in serum creatinine in the group as a whole, it was only the patients given combination treatment who had a reduction in their ascites.

More recently, Salerno et al reported that acute ISMN treatment in cirrhosis reduced diuresis and natriuresis, and reduced GFR in patients with ascites. ${ }^{32}$ However, the same study also showed that chronic administration of ISMN did not affect renal function if patients did not have ascites, but reduced diuresis and natriuresis in ascitic patients. No effect on GFR or renal plasma flow was detected.

It is clear that there are conflicting data in the literature on this subject which may be due to differences in the study populations or in the duration of treatment. Although we did not assess sodium and water excretion or GFR in this study, we found no change in RBF despite the expected changes in MAP, HVPG, and AZBF following administration of propranolol and/or ISMN. Despite the fact that all patients in our study had normal serum creatinine concentrations, it is possible that some may have had a degree of renal impairment not evident from their serum creatinine. However, when ascitic patients alone were assessed, there was still no effect on RBF. We assessed only the acute effect of these drugs on RBF, therefore we cannot draw conclusions regarding prolonged treatment. It is possible that administration of these drugs to cirrhotic patients may compromise renal function by mechanisms other than a reduction in $\mathrm{RBF}$, such as neurohumoral or tubular effects. However, the main concern of many of the above studies was a possible reduction in renal perfusion consequent on the systemic haemodynamic changes induced by these drugs.

In view of our desire to assess both $\mathrm{AZBF}$ and RBF in this study, we had to move the thermodilution catheter out of the renal vein between the two recordings of RBF. The initial position of the catheter in the left renal vein was recorded fluoroscopically and the catheter repositioned to the exact same position for the one hour recording of $\mathrm{RBF}$ using fluoroscopic guidance. To ensure validity of these measurements, three other cirrhotic patients had multiple $\mathrm{RBF}$ recordings made over one hour, with the catheter moved in and out of the left renal vein under similar conditions. The coefficient of variation in $\mathrm{RBF}$ recordings was less than $10 \%$.

Most studies assessing renal plasma flow and GFR rely on clearance of $p$-aminohippuric acid and inulin respectively, but such methods rely on high renal extraction of these substances. In the presence of sepsis or other pathological conditions leading to tissue hypoxia, microvasular abnormalities, and shunting of blood such as in cirrhosis, these methods are largely invalid. ${ }^{33}$ Although thermodilution measurement of RBF has been shown to correlate well with half the renal blood flow as calculated from $p$-aminohippuric acid infusion, ${ }^{34}$ thermodilution methods may be more accurate than clearance techniques in pathological states.

Using the thermodilution technique, we found no acute effect on RBF in patients given propranolol and/or ISMN. The possibility of a type II error exists, but the numbers studied produced significant results with regard to the other haemodynamic parameters, and particularly in the propranolol group, the patients with the lower initial RBF (whose renal function would be of most concern) actually had a rise in RBF following treatment. Although the patients given ISMN had slightly lower baseline $\mathrm{RBF}$ compared with those given propranolol or combination treatment, this difference was not significant, and as in the other therapeutic groups, these patients had no change in RBF after treatment.

In conclusion, we have detected no effect on RBF following acute administration of propranolol, ISMN, or a combination of the two drugs to cirrhotic patients, despite the anticipated changes in MAP, HVPG, and AZBF. Therefore any effect of these drugs on renal function in cirrhosis does not seem to be due to an acute reduction in $\mathrm{RBF}$.

The authors wish to thank Sister and the staff of the Department of Medicine, Royal Infirmary of Edinburgh, for their invaluable help in the completion of this study. This study was presented in part at the meeting of the European Association for the Study of the Liver, London, April 1997 (Stanley AJ, Bouchier IAD, Hayes PC. Effect of propranolol and isosorbide-5-mononitrate on renal blood flow in patients with cirrhosis. F Hepatol 1997;26(suppl 1):96A).

1 Pascal JP, Cales P, Multicenter Study Group. Propranolol in the prevention of first upper gastrointestinal tract hemorrhage in patients with cirrhosis of the liver and esophageal varices. N Engl f Med 1987;317:856-61.

2 Hayes PC, Davis JM, Lewis JA Bouchier IAD. Meta-analysis of the value of propranolol in prevention of variceal haemorrhage. Lancet 1990;1:153-6.

3 Conn HO, Grace N, Bosch J, et al and members of the Boston-New Haven-Barcelona portal hypertension study group. Propranolol in the prevention of the first variceal hemorrhage from oesophageal varices: results of a randomized double-blind cooperative clinical trial. Hepatology 1991;13:902-12.

4 Poynard T, Cales P, Pasta L, et al and the Franco-Italian Multicenter study group. $\beta$-adrenergic-antagonists in the prevention of first gastrointestinal bleeding in patients with cirrhosis and oesophan varices. An analysis of data and cirrhosis and oesophageal varices. An analysis of data and prognostic factors in 589 patients from four randomized
clinical trials. N Engl f Med 1991;324:1532-8. 5 Bernard B, Lebrec D, Mathurin P, Opolon P, Poynard T. Propranolol and sclerotherapy in the prevention of gastrointestinal rebleeding in patients with cirrhosis: a gastrointestinal rebleeding in patients
meta-analysis. $\mathcal{F}$ Hepatol $1997 ; 26: 312-24$. 
6 Hayes PC, Westaby D, Williams R. Effects and mechanisms of action of isosorbide-5-mononitrate. Gut 1988;29: of action

7 Navasa M, Chesta J, Bosch J, Rodes J. Reduction of portal pressure by isosorbide-5-mononitrate in patients with cirrhosis: effects on splanchnic and systemic hemodynanics and liver function. Gastroenterology 1989;96:1110-8.

8 Garcia-Pagan JC, Feu F, Navasa M, et al. Long-term hemodynamic effects of isosorbide-5-mononitrate in patients with cirrhosis and portal hypertension. F Hepatol 1990;11: 189-95.

9 Angelico M, Carli L, Piat C, Gentile S, Rinaldi V, Bologna E, Capocaccia L. Isosorbide-5-mononitrate versus propranolol in the prevention of first bleeding in cirrhosis. Gastroenterology 1993:104:1460-5.

10 Bertoni G, Sassatelli R, Fornaciari G, et al. Oral isosorbide5 -mononitrate reduces the rebleeding rate during the course of injection sclerotherapy for esophageal varices. course of injection sclerotherapy for

11 Garcia-Pagan JC, Feu F, Bosch J, Rodes J. Propranolol compared to propranolol plus isosorbide-5-mononitrate for portal hypertension: a randomis

12 Vorobioff J, Picabea E, Gamen M, et al. Propranolol compared with propranolol plus isosorbide dinitrate in portal-hypertensive patients: long-term hemodynamic and renal effects. Hepatology 1993;18:477-84

13 Rector WG Jr, Reynolds TB. Propranolol in the treatment on cirrhotic ascites. Arch Intern Med 1984;144:1761-3.

14 Ljubicic N, Bilic A, Plavsic V. Effect of propranolol on urinary prostaglandin E2 excretion and renal interlobar arterial blood flow after furosemide administration in patients with hepatic cirrhosis. Eur 7 Clin Pharmacol 1992;43: 555-8.

15 Bolognesi M, Sacerdoti D, Merkel C, Gatta A. Duplex Doppler sonographic evaluation of splanchnic and renal effects of single agent and combined therapy with nadolol and isosorbide-5-mononitrate in cirrhotic patients. F Ultraand isosorbide-

16 Salmeron JM, Ruiz del Arbol L, Gines A, et al. Renal effects of acute isosorbide-5-mononitrate administration in cirof acute isosorbide-5-mononitrate
rhosis. Hepatology 1993;17:800-6.

17 Henriksen JH, Ring-Larsen H. Renal effects of drugs used in the treatment of portal hypertension. Hepatology 1993;18:688-95.

18 Hayes PC, Terrace D, Peaston I, Bouchier IAD, Redhead DN, Brash HM. Computerised system for the continuous measurement of azygos venous blood flow. Gut 1992;33: 372-4.

19 Epstein $M$, ed. The kidney in liver disease. Baltimore: Williams \& Wilkins, 1988:1-656.

20 Wilkinson R. $\beta$-Blockers and renal function. Drugs 1982;23; 195-206.
21 Henriksen JH, Bendtsen F, Gerbes AL, Christensen NJ, Ring-Larsen H, Sorensen TIA. Estimated central blood
volume in cirrhosis: relationship to sympathetic nervous volume in cirrhosis: relationship to sympathetic nervous activity, beta-adrenergic blockade
tor. Hepatology 1992;16:1163-70.

22 Best JH, Halter JB. Blood pressure and norepinephrine pillover during propranolol infusions in humans. $A m \mathcal{F}$ Physiol 1985;248:R400-6.

23 Bernardi M, De Palma R, Trevisani F, et al. Renal function and effective $\beta$-blockade in cirrhosis with ascites. 7 Hepatol 1989;8:279-86.

24 Bataille C, Bercoff E, Pariente EA, Valla D, Lebrec D. Effects of propranolol on renal blood flow and renal function in patients with cirrhosis. Gastroenterology 1984;86: tion in p

25 Wilkinson SR, Bernardi M, Smith IK, Jowet TP, Slater JDH, Williams R. Effect of $\beta$-adrenergic blockade on the renin-angiotensin system, sodium excretion, and renal hemodynamics in cirrhosis with ascites. Gastroenterology 1977;73:659-63.

26 Hayes PC, Stewart WW, Bouchier IAD. Influence of propranolol on weight and salt and water homeostasis in chronic liver disease. Lancet 1984;ii:1064-8.

27 Garcia-Tsao G, Grace ND, Groszman RJ, et al. Short-term effects of propranolol on portal pressure. Hepatology 1986; 6:101-6.

28 D'Amico G, Pagliaro L, Bosch J. The treatment of portal hypertension: a meta-analytic review. Hepatology 1995;22: 332-51.

29 Merkel C, Finucci G, Zuin R. Effects of isosorbide dinitrate on portal hypertension in alcoholic cirrhosis. 7 Hepatol 1987;4:174-80

30 Morrillos RM, Planas R, Cabre E, et al. Propranolol plus isosorbide-5-mononitrate for portal hypertension in cirrhosis: long-term hemodynamic and renal effects. Hepatology 1994;20:1502-8.

31 Merkel C, Gatta A, Donada C, et al and the GTIP. Long-term effect of nadolol or nadolol plus isosorbide-5mononitrate on renal function and ascites formation in patients with cirrhosis. Hepatology 1995;22:808-13.

32 Salerno F, Borroni G, Lorenzano E, et al. Long-term administration of isosorbide-5-mononitrate does not impair renal function in cirrhotic patients. Hepatology 996;23:1135-40.

33 Brenner M, Schaer GL, Mallory DL, Suffredinin AF, Parrillo JE. Detection of renal blood flow abnormalities in septic and critically ill patients using a newly designed indwelling thermodilution renal vein catheter. Chest 1990;98: $170-9$.

34 Hornych A, Brod J, Slechta V. The measurement of renal venous outflow in man by the local thermodilution method. Nephron 1971;8:17-32. 